\title{
Minireview
}

\section{Tumours and tremors: how PTEN regulation underlies both}

\author{
RH Kim ${ }^{1,2,3}$ and TW Mak ${ }^{*, 1,2,3}$ \\ 'Campbell Family Institute for Breast Cancer Research, Toronto, Ontario M5G 2CI, Canada; ${ }^{2}$ Ontario Cancer Institute, Toronto, Ontario M5G $2 \mathrm{Cl}$ \\ Canada; ${ }^{3}$ Department of Medical Biophysics, University of Toronto, Toronto, Ontario M5G 2CI, Canada
}

Mutations of the tumour suppressor PTEN (phosphatase and tensin homolog deleted on chromosome 10) are seen in many human cancers. However, dysregulation of PTEN may be involved in other disease states such as Parkinson's disease. This minireview describes recent work examining PTEN regulation and its implications for the development of both cancer and neurodegenerative disease.

British Journal of Cancer (2006) 94, 620-624. doi:I0.1038/sj.bjc.6602994 www.bjcancer.com

Published online 2I February 2006

(c) 2006 Cancer Research UK

Keywords: PTEN; DJ-I; tumour suppression; Parkinson's disease

\section{PTEN AND TUMORIGENESIS}

Almost a decade has passed since the discovery of the lipid phosphatase PTEN (phosphatase and tensin homolog deleted on chromosome 10) as an important tumour suppressor. The PTEN gene was originally identified by three independent groups using different methodologies: a representational difference analysis of tumours; a high-density scan of chromosome 10q in glioma cell lines and a screening of human cDNA libraries designed to detect protein phosphatases upregulated in response to transforming growth factor $\beta$ (TGF- $\beta$ ) (Maehama et al, 2001). Mutations and deletions in the PTEN locus were subsequently found to be associated with a broad range of human cancers. While mutations occur throughout the length of the PTEN gene, tumorigenic alterations are clustered in exon 5 encoding the catalytic domain, confirming that the PTEN lipid phosphatase activity is critical for tumour suppression (Ali et al, 1999).

Loss of heterozygosity (LOH) studies comparing early and advanced cancers suggest that PTEN may play its most important role in advanced cancers of particular tissues (Ali et al, 1999). For example, the mutation frequency of PTEN is high in malignant glial tumours, but much more modest in lower grade gliomas and glioneuronal tumours. Furthermore, alterations of PTEN in glial tumours are associated with shorter median survival. Prostate tumours are associated with LOH of $10 \mathrm{q} 23$, with mutations of $P T E N$ reported most frequently in metastatic disease and in cases with a poor prognosis. Although second mutations of PTEN occur less frequently than $\mathrm{LOH}$, loss of PTEN protein occurs frequently in high grade and Gleason score prostate tumours. $P T E N$ is also considered as the most frequently mutated gene in endometrial cancers. Indeed, mutation of PTEN likely contributes to the initiation of endometrial tumorigenesis, as this gene is

\footnotetext{
*Correspondence: Dr TW Mak, Campbell Family Institute for Breast Cancer Research, 620 University Avenue, Toronto, Ontario M5G 2Cl, Canada; E-mail: tmak@uhnres.utoronto.ca

Received 6 October 2005; revised 23 January 2006; accepted 23 January 2006; published online 21 February 2006
}

also altered in premalignant endometrial hyperplasia (Sansal and Sellers, 2004).

Germline mutations in tumour suppressor genes are associated with cancer predisposition syndromes that result in the appearance of hamartomas (hyperplastic and disorganised growths) throughout the body. Germline mutations of the PTEN gene are found in two hamartoma disorders called Cowden syndrome (MIM 158350) and Bannayan-Riley-Ruvalcaba (MIM 153480) syndrome. Cowden syndrome is a rare, autosomal dominant, familial cancer syndrome characterised by hamartomas, multiple smooth facial papules, acral keratosis and multiple oral papillomas. In contrast, Bannayan-Riley-Ruvalcaba syndrome is marked by macrocephaly, lipomatosis, hemangiomatosis and speckled penis (Eng, 2003). As with sporadic tumours, the germline PTEN mutations associated with these hamartoma syndromes are clustered around the catalytic site (Ali et al, 1999).

Enhanced tumorigenesis has also been observed in Ptendeficient mice. Numerous conventional and conditional genetargeting murine models of pten deficiency have been generated to investigate the physiological functions of PTEN. Conventional gene-targeting of pten in all mouse tissues $\left(\mathrm{pten}^{-1-}\right.$ mice) results in developmental delay and lethality at embryonic days 6.5-8.5 due to a failure in chorio-allantoic fusion (Kishimoto et al, 2003). However, pten ${ }^{+I-}$ mice eventually develop LOH of the remaining pten allele, leading to the appearance of tumours in the endometrium, liver, prostate, gastrointestinal tract, thyroid and thymus. Interestingly, in addition to tumours, tissue-specific deletion of pten can result in hyperplasia, autoimmunity, glucose dysregulation or neurological deficits (Kishimoto et al, 2003).

\section{STRUCTURE OF THE PTEN GENE}

The human genomic PTEN locus consists of nine exons encoding a $5.5 \mathrm{~kb}$ mRNA that specifies a 403 amino-acid open reading frame. The translation product is a $53 \mathrm{kDa}$ protein with extensive homology to tensin/auxilin and protein tyrosine phosphatases (PTPs). Studies of the crystal structure of PTEN have revealed an enlarged catalytic site (relative to other PTPs) that accommodates 
large phosphoinositide substrates, and a C2 domain that mediates the recruitment of proteins to the membrane (Maehama et al, 2001). In addition to the $C 2$ domain, the C-terminus of PTEN contains a PSD95/SAP90, DLG, ZO-1 (PDZ)-binding domain (involved in protein-protein interactions) and two proline, glutamic acid, serine and threonine (PEST) sequences (involved in protein degradation) (Leslie and Downes, 2004).

\section{FUNCTIONS OF THE PTEN PROTEIN}

The N-terminus of the PTEN protein is similar to that of PTPs, and PTEN was initially thought to be a dual specificity PTP. Indeed, the PTP activity of PTEN has been implicated in cellular processes such as cell motility (Raftopoulou et al, 2004). However, PTEN removes phosphate groups from phosphorylated threonine and serine protein targets only very inefficiently, and its lipid phosphatase activity is now considered its most important attribute. PTEN is most active against negatively charged peptides, and its primary cellular substrate is the membrane lipid phosphoinositide-3,4,5-trisphosphate ( $\mathrm{PIP}_{3}$ ) (Maehama et al, 2001). Phosphatidylinositol 3,4,5-trisphosphate is the principal second messenger of the phosphoinositide- 3 kinase $\left(\mathrm{PI}^{\prime} \mathrm{K}\right)$ pathway that mediates receptor tyrosine kinase signalling through to the survival kinase PKB/Akt (Chan et al, 1999). Increased levels of $\mathrm{PIP}_{3}$ at the membrane cause $\mathrm{PH}$ domain-containing proteins such as PKB/Akt and PDK-1 to colocalise, resulting in PDK-1-mediated phosphorylation and activation of $\mathrm{PKB} / \mathrm{Akt}$. Activated $\mathrm{PKB} / \mathrm{Akt}$ transfers a phosphate group to target proteins involved in cell survival, cell cycling and metabolism (Chan et al, 1999). PTEN exerts its tumour-suppressive effect by dephosphorylating $\mathrm{PIP}_{3}$, thereby negatively regulating $\mathrm{PKB} /$ Akt activation and the $\mathrm{PI}^{\prime} \mathrm{K}$ survival pathway (Maehama et al, 2001).

PTEN-mediated modulation of $\mathrm{PI}^{\prime} \mathrm{K}$ signalling plays a key role in regulating cellular functions associated with proliferation/cell cycle, programmed cell death (PCD), angiogenesis and migration. Engineered overexpression of PTEN induces profound growth suppression primarily by promoting cell cycle arrest. For example, PTEN overexpression leads to G1 arrest in glioma, breast, endometrial and prostate cancer cells (Maehama et al, 2001). This cell cycle arrest requires PTEN's lipid phosphatase activity, can be enhanced by low serum, and can be rescued with the introduction of constitutively active forms of $\mathrm{PI} 3^{\prime} \mathrm{K}, \mathrm{PKB} / \mathrm{Akt}$ or PDK-1 (Maehama et al, 2001). One mechanism by which PTEN induces cell cycle arrest is by regulating $\mathrm{PKB} / \mathrm{Akt}$ such that levels of the cell cycle inhibitor p $27^{\mathrm{kip} 1}$ are increased. Overexpression of PTEN also correlates with decreased total levels and nuclear localisation of cyclin D1, a key cell cycle molecule regulated by PKB/Akt. Other $\mathrm{PKB} /$ Akt-regulated cell cycle mediators affected by PTEN expression levels include the forkhead transcription factors and glycogen synthase kinase (Maehama et al, 2001).

In addition to regulating the cell cycle, PTEN controls various forms of PCD. Overexpression of PTEN induces the apoptosis of many different cell types, and pten-deficient murine embryonic fibroblasts (MEFs) are resistant to various apoptotic stimuli (Maehama et al, 2001). PTEN's promotion of the apoptotic response may cooperate with PTEN's function in cell cycle arrest, and may be mediated by more than one mechanism. Through its effects on PKB/Akt, PTEN can induce the activation of proapoptotic molecules such as Fas and bim, while promoting the inactivation of antiapoptotic molecules such as the bcl-2 family member Bad and the X-linked inhibitor of apoptosis (XIAP) that blocks caspase activation. As a result, pten ${ }^{+1-}$ mice are abnormally susceptible to Fas-mediated apoptosis, and ectopic expression of PTEN sensitises glioblastoma cells to irradiationand Fas-induced apoptosis characterised by increased caspase- 3 activity (Kishimoto et al, 2003). PTEN also plays an important role in the induction of death in cells that lose contact with the extracellular matrix, a type of PCD called anoikis. Reconstitution of PTEN in PTEN-deficient cells restores anoikis by negatively regulating the scaffold protein focal adhesion kinase (FAK). A detectable decrease in FAK phosphorylation is also observed when PTEN overexpression results in inhibition of fibronectin-induced formation of actin stress fibres and prevention of cell spreading, migration on extracellular matrix proteins and invasion. Conversely, an absence of PTEN function may allow unregulated cell spreading and invasion that could contribute to metastasis (Maehama et al, 2001).

In addition to promoting the survival and metastasis of tumour cells, PTEN mutations can contribute to malignancy through altered regulation of protein synthesis and cellular nutrient consumption. Through studies using the fruitfly Drosophila melanogaster, it has been shown that PTEN inhibits the target of rapamycin (mTOR) metabolic pathway. Dysregulation of this pathway resulted in altered protein translation and amino-acid consumption, which in turn led to marked increases in individual cell size and organ size. As well, recent studies have suggested that PTEN mutations can influence the activity of the vascular endothelial-like growth factor (VEGF) that stimulates blood vessel formation. Mutation of PTEN can destabilise hypoxiainducible transcription factor 1 (HIF-1), the molecule that drives VEGF transcription. Thus, in the absence of PTEN, a combination of increased metabolism and enhanced angiogenesis may promote tumour cell growth and metastasis (Sansal and Sellers, 2004).

\section{REGULATION OF THE PTEN PROTEIN}

Some tumours, such as sporadic breast cancers, contain wild-type PTEN genes but decreased PTEN protein immunoreactivity. This observation implies that dysregulation of a normal PTEN gene or protein can abrogate PTEN function and lead to neoplastic disease. $P T E N$ was initially cloned as a gene whose transcription was downregulated by TGF- $\beta$. However, in addition to transcriptional regulation, the function of the normal PTEN protein can be modulated by protein-protein interactions, phosphorylation and other epigenetic factors.

The C-terminus of PTEN is critical for its tumour suppressive activity and contains putative phosphorylation sites thought to play a role in PTEN regulation (Leslie and Downes, 2004). Residues Ser380, Thr382 and Thr383 are substrates for phosphorylation by casein kinase II (CK2). Casein kinase II-mediated phosphorylation of these residues alters electrostatic shielding and decreases the affinity of the catalytic and C2 domains of PTEN for the membrane, decreasing PTEN activity. Casein kinase II-mediated phosphorylation also stabilises the PTEN protein by preventing its proteasomal degradation and proteolysis by caspases, while keeping it in an inactive state. Inhibition of CK2-mediated PTEN phosphorylation results in increased PTEN activity and a corresponding reduction in $\mathrm{PKB} / \mathrm{Akt}$ activation. This activation of unphosphorylated PTEN may be due to a conformational change that opens the PDZ-binding sites. The presence of open PDZ-binding sites in PTEN allows interactions with PDZcontaining proteins such as membrane-associated guanylate kinase inverted-2 (MAGI-2), as well as the formation of active PTEN-associated complexes that can decrease PKB/Akt activation (Leslie and Downes, 2004). Overexpression of MAGI-2 has been shown to restore PTEN stability in vinculin null F9 cells, which are cells that contain decreased PTEN protein but normal levels of PTEN mRNA (Subauste et al, 2005). Other PDZ domain-containing proteins that interact with PTEN include MAGI-3, hDLG (discslarge) and hMAST (microtubule-associated serine-threonine kinase), all of which can bind to PTEN and affect its stability through phosphorylation of the C-terminal tail (Leslie and Downes, 2004; Valiente et al, 2005). 
Another PTEN-binding protein, thioredoxin, is a redox protein that is overexpressed in a large number of tumours. Cys32 of thioredoxin forms a disulphide bond with Cys212 in the C2 domain of PTEN, inhibiting PTEN activity (Meuillet et al, 2004). PTEN can also be reversibly inactivated by exposure to hydrogen peroxide, which induces the formation of an internal disulphide bond between Cys71 and Cys124 in the catalytic core (Lee et al, 2002). This type of PTEN inactivation is seen in cell cultures following growth factor-induced peroxide production, suggesting that redox inactivation of PTEN is a physiologic response to mitogen stimulation (Kwon et al, 2004). Consistent with this hypothesis, increases in hydrogen peroxide specifically induced by mitochondrial dysfunction lead to the oxidation and inactivation of PTEN and increased $\mathrm{PI} 3^{\prime} \mathrm{K}$ signalling (Connor et al, 2005). The ability of oxidised PTEN to regain its tumour suppressive activity depends on the capacity of thioredoxin to reduce the inactive form, and a total loss of PTEN function can occur if the redox status of the cell is abnormal (Lee et al, 2002; Kwon et al, 2004).

At the mRNA level, analyses of various tumours have shown that methylation of the PTEN promoter can result in transcriptional silencing of the PTEN gene (Kang et al, 2002). Interestingly, the function of the PTEN promoter and PTEN mRNA expression appear to be regulated by more than one transcription factor. For example, the genomic PTEN promoter sequence contains a GC-rich $5^{\prime}$ untranslated region that can be activated by the transcription factor Sp1 to drive constitutive PTEN expression (Han et al, 2003). Another transcription factor, early growth response-1 (Egr-1), binds to the PTEN untranslated region and activates PTEN transcription during irradiation-induced signalling. The PTEN promoter also contains binding sites for another irradiationsensitive transcription factor, p53, which has been shown to increase levels of both PTEN mRNA and protein (Leslie and Downes, 2004). NF- $\kappa$ B increases PTEN expression during cellular differentiation but decreases PTEN transcription in cells responding to apoptotic stimuli, suggesting that NF- $\kappa \mathrm{B}$-induced transcription of PTEN may depend on the cellular context. Finally, a ligandactivated nuclear receptor called peroxisome proliferator-activated receptor $(\operatorname{PPAR} \gamma)$ is involved in anti-inflammatory responses thought to be mediated by transcriptionally controlled upregulation of PTEN (Leslie and Downes, 2004).

\section{USE OF D. MELANOGASTER TO INVESTIGATE PTEN SIGNALLING}

The $\mathrm{PI} 3^{\prime} \mathrm{K}$ signalling pathway regulated by PTEN is largely conserved in metazoans, and PTEN and $\mathrm{PI}^{\prime} \mathrm{K}$ homologues have been identified in many species. In addition to its effect on translation, much has been learned about the biological functions of PTEN and the $\mathrm{PI}^{\prime} \mathrm{K}$ pathway from the fruit fly, D. melanogaster. Drosophila $\mathrm{PI}^{\prime} \mathrm{K}$ signalling involves homologues of insulin receptor ligand (CHICO), the insulin/IGF-1 receptor (DInr), $\mathrm{PI}^{\prime} \mathrm{K}$ (Dp110), PTEN (dPTEN) and PKB/Akt (dPKB/dAkt1) (Simpson and Parsons, 2001). Mutation of any one of the genes encoding these proteins produces an activation of $\mathrm{PI}^{\prime} \mathrm{K}$ signalling that drives cellular expansion and proliferation and leads to increases in cell size, cell number and organ size. Conversely, overexpression of dPTEN or dominant-negative $\mathrm{dPI}^{\prime} \mathrm{K}$ in Drosophila embryos triggers apoptosis similar to that observed in dPKB/dAkt1- or dPDK1-deficient flies. In the Drosophila eye, PTEN overexpression inhibits cell cycle progression and promotes cell death (Maehama et al, 2001). In our own work, we used a system based on this latter observation to isolate dPTEN regulators in a gain-of-function screening protocol employing a P-element library. We identified $D J-1$, a putative oncogene involved in autosomal recessive earlyonset Parkinson's disease (PD) (Bonifati et al, 2003), as a novel suppressor of PTEN function (Kim et al, 2005a).

\section{LINKS BETWEEN DJ-1 AND TUMORIGENESIS}

Several lines of evidence suggest that DJ-1 plays a role in human tumorigenesis. Breast cancer patients have elevated levels of serum DJ-1 and circulating anti-DJ-1 autoantibodies compared to healthy and non-breast cancer patients (Le Naour et al, 2001). Furthermore, DJ-1 protein is increased in primary non-small-cell lung carcinoma samples (MacKeigan et al, 2003). As with prostate cancer, the rate of $\mathrm{LOH}$ at $10 \mathrm{q} 23$ in the lung and breast cancers is greater than the rate of mutation of the remaining PTEN allele. This observation suggests that, in these cases, epigenetic regulation of PTEN has caused its loss of function. Although the underlying mechanism has yet to be clearly defined, it is quite possible that faulty control of a PTEN regulator such as DJ-1 is responsible. In lung cancer patients, an elevation in $D J-1$ transcripts correlates with a poor prognosis, particularly for Stage I disease (Kim et al, 2005a). Higher levels of DJ-1 mRNA are also present in patients lacking mutations in Ras, a well-known molecular marker of tumorigenesis. These data suggest that DJ-1 is important for tumour initiation and may be a useful prognostic marker in certain types of lung cancer. In primary breast cancer samples, DJ-1 expression correlates positively with phospho-PKB/Akt immunoreactivity. In vitro, DJ-1 inhibits PTEN's negative regulation of the $\mathrm{PI}^{\prime} \mathrm{K}$ pathway and increases $\mathrm{PKB} / \mathrm{Akt}$ activation. Thus, DJ-1 is likely oncogenic because of its negative regulatory effects on PTEN, effects that indirectly promote activation of the $\mathrm{PI}^{\prime} \mathrm{K}$ cell survival pathway.

\section{LINKS BETWEEN PTEN, DJ-1 AND PD}

Parkinson's disease and cancer are two pathologic processes resulting from excessive signalling by one of two sets of opposing forces: those driving cell death and those promoting cell survival. Parkinson's disease results from the excessive death of dopaminergic neurons in the substantia nigra pars compacta (SNpc) in the brain, while tumorigenesis is driven by excessive cell survival in the target tissue. The underlying signalling mechanisms of cell death and survival can be generalised and applied to most cell types and tissues, implying that the balance can be shifted from death to survival and vice versa by aberrations of the same signalling pathway. Early epidemiological studies showed a decreased incidence of cancer in PD patients, later confirmed in larger studies (West et al, 2005). Moreover, the genes associated with familial forms of PD (the PARK loci) are now implicated in tumorigenesis. For example, $\alpha$-synuclein (SNCA; PARK1) expression is upregulated in glioma cell lines, schwannomas, medulloblastomas and breast and ovarian carcinomas. Deletions of Parkin (PARK2) have been identified in hepatocellular carcinomas and in breast, ovarian and non-small-cell lung cancers. Finally, ubiquitin C-terminal hydrolase (UCH-L1; PARK5) is overexpressed in oesophageal and squamous cell carcinomas, and in pancreatic and colorectal cancers (West et al, 2005). The effects of PARK mutations on tumorigenesis are unclear and, due to the unknown functions of these PD-associated genes, conclusions about the nature of these mutations (gain-of-function $v s$ loss-of-function) are difficult to establish. Moreover, loss/gain-of-function mutations in PD may have additional distinct effects on tumorigenesis, confounding attempts to 'pigeon-hole', a particular gene. Nevertheless, genes associated with familial PD have been shown to regulate cell death and/or the cell cycle, and several lines of evidence imply that malfunction of a shared biochemical pathway may lead to PD or cancer. Firstly, during tumorigenesis, the degradation of $\mathrm{p} 27^{\mathrm{kip} 1}$ can be driven by either PTEN or UCH-L1 (Caballero et al, 2002; Viglietto et al, 2002). Secondly, deletions of the Parkin gene in Drosophila result in PKB/Akt activation (Yang et al, 2005). Thirdly, PINK1, which encodes a kinase downregulated in the absence of PTEN, has been identified as the sixth locus (PARK6) associated with familial PD (West et al, 2005). 
A key link between PTEN dysregulation and PD may be DJ-1, isolated originally as PARK7. The identification of loss-of-function mutations in the DJ-1 gene in PARK7 PD families is consistent with the notion that DJ-1 acts to promote neuron survival via the $\mathrm{PI}^{\prime} \mathrm{K}$ signalling pathway. The biochemistry of the neurodegeneration in $\mathrm{PD}$ points to mitochondrial oxidative stress as the mechanism driving neuronal death in the SNpc. In Drosophila, an siRNAinduced reduction of DJ-1 causes neurons to become hypersensitive to oxidative stress, and these flies show neurodegeneration that is exacerbated by coexpression of PTEN (Yang et al, 2005). Significantly, this hypersensitivity to oxidative stress is also seen in the neurons of $d j$-1-deficient mice (Kim et al, 2005b). In both organisms, the increased susceptibility to oxidative stress can be rescued by activation of the $\mathrm{PI} 3^{\prime} \mathrm{K}$ pathway (Kim et al, 2005b; Meulener et al, 2005; Yang et al, 2005). Other work has implicated DJ-1 as a sensor of reactive oxygen species and as a molecular chaperone (Shendelman et al, 2004), and DJ-1 may be able to influence the protein stability or oxidative state of elements of the PI3'K pathway. For example, PTEN and ASK1, a component of a DJ-1-containing complex (Junn et al, 2005), are both regulated by thioredoxin (Meuillet et al, 2004). These findings suggest that DJ-1 may act in cooperation with redox-sensitive proteins to regulate its substrates, and that DJ-1-mediated regulation of one of these substrates, PTEN, plays a vital role in preventing both cancer and neurodegenerative disease.

\section{CONCLUSIONS AND PERSPECTIVES}

The tumour suppressor gene PTEN is currently recognised as one of the most frequently mutated genes in human cancers. As befits a critical gene, PTEN is regulated at multiple levels, including transcription, protein stability and phosphorylation. Understanding this regulation is crucial for the effective design of novel cancer therapeutics. Inhibitors of the PI $3^{\prime} \mathrm{K}$ pathway such as wortmannin and LY294002 are commonly employed in the research laboratory, while receptor tyrosine kinase inhibitors such as gefitinib (Iressa; AstraZeneca, Wilmington, DE, USA), trastuzumab (Herceptin; Genentech, San Francisco, CA, USA) and imatinib (Gleevec;

\section{REFERENCES}

Ali IU, Schriml LM, Dean M (1999) Mutational spectra of PTEN/MMAC1 gene: a tumor suppressor with lipid phosphatase activity. J Natl Cancer Inst 91: $1922-1932$

Bonifati V, Rizzu P, van Baren MJ, Schaap O, Breedveld GJ, Krieger E, Dekker MC, Squitieri F, Ibanez P, Joosse M, van Dongen JW, Vanacore N, van Swieten JC, Brice A, Meco G, van Duijn CM, Oostra BA, Heutink P (2003) Mutations in the DJ-1 gene associated with autosomal recessive early-onset parkinsonism. Science 299: 256-259

Caballero OL, Resto V, Patturajan M, Meerzaman D, Guo MZ, Engles J, Yochem R, Ratovitski E, Sidransky D, Jen J (2002) Interaction and colocalization of PGP9.5 with JAB1 and p27(Kip1). Oncogene 21: $3003-3010$

Chan TO, Rittenhouse SE, Tsichlis PN (1999) AKT/PKB and other D3 phosphoinositide-regulated kinases: kinase activation by phosphoinositide-dependent phosphorylation. Annu Rev Biochem 68: 965-1014

Connor KM, Subbaram S, Regan KJ, Nelson KK, Mazurkiewicz JE, Bartholomew PJ, Aplin AE, Tai YT, Aguirre-Ghiso J, Flores SC, Melendez JA (2005) Mitochondrial $\mathrm{H}_{2} \mathrm{O}_{2}$ regulates the angiogenic phenotype via PTEN oxidation. J Biol Chem 280: 16916-16924. Epub 2005 Feb 8

Eng C (2003) PTEN: one gene, many syndromes. Hum Mutat 22: 183-198

Han B, Dong Z, Liu Y, Chen Q, Hashimoto K, Zhang JT (2003) Regulation of constitutive expression of mouse PTEN by the $5^{\prime}$-untranslated region. Oncogene 22: $5325-5337$

Junn E, Taniguchi H, Jeong BS, Zhao X, Ichijo H, Mouradian MM (2005) Interaction of DJ-1 with Daxx inhibits apoptosis signal-regulating kinase 1 activity and cell death. Proc Natl Acad Sci USA 102: 9691 -9696. Epub 2005 Jun 27
Novartis, Summit, NJ, USA) have been added to the clinical armamentarium used to treat certain types of human cancers. Inhibitors of downstream effectors of the $\mathrm{PI}^{\prime} \mathrm{K}$ pathway, such as the rapamycin derivatives CCI-779 (Wyeth Research, Madison, NJ, USA) and RAD001 (Novartis) are under investigation to determine their efficacy as mTOR inhibitors in tumours. Similarly, because oxidative stress and reactive oxygen species can inactivate PTEN, alternative therapeutics that target PTEN's redox status are in the development phase.

The involvement of the $\mathrm{PI} 3^{\prime} \mathrm{K}$ pathway and putative regulators of PTEN (such as DJ-1) in PD indicates that the importance of PTEN regulation extends beyond the known roles of this gene in tumorigenesis, hamartomatous syndromes and autoimmunity. As PTEN is regulated by phosphorylation and degradation via the ubiquitin-proteasome system, proteasomal targeting may offer a potential therapeutic modality for the treatment of PTEN-related cancers. For example, the proteasome inhibitor bortezomib (Velcade; Millennium Pharmaceuticals, Cambridge, MA, USA) has been effective in treating multiple myeloma because survival factors that depend on proteasomal degradation (such as NF-êB) are inactivated. However, caution is warranted in using this approach, as other proteasome inhibitors induce Parkinsonian-like symptoms when administered to rats (McNaught et al, 2004). Intriguingly, this latter finding offers additional support for the hypothesis that cancer and PD may both result from PTEN dysregulation. All these developments point towards exciting new avenues for the complete elucidation of PTEN's physiological functions, and the manipulation of this knowledge for the treatment of both tumorigenesis and neurodegeneration.

\section{ACKNOWLEDGEMENTS}

We are very grateful to Mary Saunders for scientific editing of the manuscript. RHK is a recipient of $\mathrm{MD} / \mathrm{PhD}$ scholarships from the CIHR. RHK is also supported by the Frank Fletcher Memorial Fund, David Rae Scholarship, Paul Starita Fellowship and Edward Ketchum Graduate Student Scholarship.
Kang YH, Lee HS, Kim WH (2002) Promoter methylation and silencing of PTEN in gastric carcinoma. Lab Invest 82: 285-291

Kim RH, Peters M, Jang Y, Shi W, Pintilie M, Fletcher GC, DeLuca C, Liepa J, Zhou L, Snow B, Binari RC, Manoukian AS, Bray MR, Liu FF, Tsao MS, Mak TW (2005a) DJ-1, a novel regulator of the tumor suppressor PTEN. Cancer Cell 7: 263-273

Kim RH, Smith PD, Aleyasin H, Hayley S, Mount MP, Pownall S, Wakeham A, You-Ten AJ, Kalia SK, Horne P, Westaway D, Lozano AM, Anisman H, Park DS, Mak TW (2005b) Hypersensitivity of DJ-1-deficient mice to 1-methyl-4-phenyl-1,2,3,6-tetrahydropyrindine (MPTP) and oxidative stress. Proc Natl Acad Sci USA 102: 5215-5220. Epub 2005 Mar 22

Kishimoto H, Hamada K, Saunders M, Backman S, Sasaki T, Nakano T, Mak TW, Suzuki A (2003) Physiological functions of Pten in mouse tissues. Cell Struct Funct 28: 11-21

Kwon J, Lee SR, Yang KS, Ahn Y, Kim YJ, Stadtman ER, Rhee SG (2004) Reversible oxidation and inactivation of the tumor suppressor PTEN in cells stimulated with peptide growth factors. Proc Natl Acad Sci USA 101: 16419-16424. Epub 2004 Nov 8

Le Naour F, Misek DE, Krause MC, Deneux L, Giordano TJ, Scholl S, Hanash SM (2001) Proteomics-based identification of RS/DJ-1 as a novel circulating tumor antigen in breast cancer. [comment]. Clin Cancer Res 7: $3328-3335$

Lee SR, Yang KS, Kwon J, Lee C, Jeong W, Rhee SG (2002) Reversible inactivation of the tumor suppressor PTEN by $\mathrm{H}_{2} \mathrm{O}_{2}$. J Biol Chem 277: 20336-20342. Epub 2002 Mar 26

Leslie NR, Downes CP (2004) PTEN function: how normal cells control it and tumour cells lose it. Biochem J 382: 1-11 
MacKeigan JP, Clements CM, Lich JD, Pope RM, Hod Y, Ting JP (2003) Proteomic profiling drug-induced apoptosis in non-small cell lung carcinoma: identification of RS/DJ-1 and RhoGDIalpha. Cancer Res 63: $6928-6934$

Maehama T, Taylor GS, Dixon JE (2001) PTEN and myotubularin: novel phosphoinositide phosphatases. Annu Rev Biochem 70: $247-279$

McNaught KS, Perl DP, Brownell AL, Olanow CW (2004) Systemic exposure to proteasome inhibitors causes a progressive model of Parkinson's disease. Ann Neurol 56: $149-162$

Meuillet EJ, Mahadevan D, Berggren M, Coon A, Powis G (2004) Thioredoxin-1 binds to the C2 domain of PTEN inhibiting PTEN's lipid phosphatase activity and membrane binding: a mechanism for the functional loss of PTEN's tumor suppressor activity. Arch Biochem Biophys 429: $123-133$

Meulener M, Whitworth AJ, Armstrong-Gold CE, Rizzu P, Heutink P, Wes PD, Pallanck LJ, Bonini NM (2005) Drosophila DJ-1 mutants are selectively sensitive to environmental toxins associated with Parkinson's disease. Curr Biol 15: 1572 - 1577

Raftopoulou M, Etienne-Manneville S, Self A, Nicholls S, Hall A (2004) Regulation of cell migration by the $\mathrm{C} 2$ domain of the tumor suppressor PTEN. Science 303: 1179-1181

Sansal I, Sellers WR (2004) The biology and clinical relevance of the PTEN tumor suppressor pathway. J Clin Oncol 22: 2954-2963
Shendelman S, Jonason A, Martinat C, Leete T, Abeliovich A (2004) DJ-1 is a redox-dependent molecular chaperone that inhibits alpha-synuclein aggregate formation. PLoS Biol 2: e362. Epub 2004 Oct 05

Simpson L, Parsons R (2001) PTEN: life as a tumor suppressor. Exp Cell Res 264: $29-41$

Subauste MC, Nalbant P, Adamson ED, Hahn KM (2005) Vinculin controls PTEN protein level by maintaining the interaction of the adherens junction protein beta-catenin with the scaffolding protein MAGI-2. J Biol Chem 280: 5676-5681. Epub 2004 Dec 5

Valiente M, Andres-Pons A, Gomar B, Torres J, Gil A, Tapparel C, Antonarakis SE, Pulido R (2005) Binding of PTEN to specific PDZ domains contributes to PTEN protein stability and phosphorylation by MAST kinases. J Biol Chem 10: 10

Viglietto G, Motti ML, Bruni P, Melillo RM, D’Alessio A, Califano D, Vinci F, Chiappetta G, Tsichlis P, Bellacosa A, Fusco A, Santoro M (2002) Cytoplasmic relocalization and inhibition of the cyclin-dependent kinase inhibitor p27(Kip1) by $\mathrm{PKB} / \mathrm{Akt}$-mediated phosphorylation in breast cancer. Nat Med 8: 1136-1144. Epub 2002 Sep 16

West AB, Dawson VL, Dawson TM (2005) To die or grow: Parkinson's disease and cancer. Trends Neurosci 28: 348-352

Yang Y, Gehrke S, Haque ME, Imai Y, Kosek J, Yang L, Beal MF, Nishimura I, Wakamatsu K, Ito S, Takahashi R, Lu B (2005) Inactivation of Drosophila DJ-1 leads to impairments of oxidative stress response and phosphatidylinositol 3-kinase/Akt signaling. Proc Natl Acad Sci USA 9: 9 